
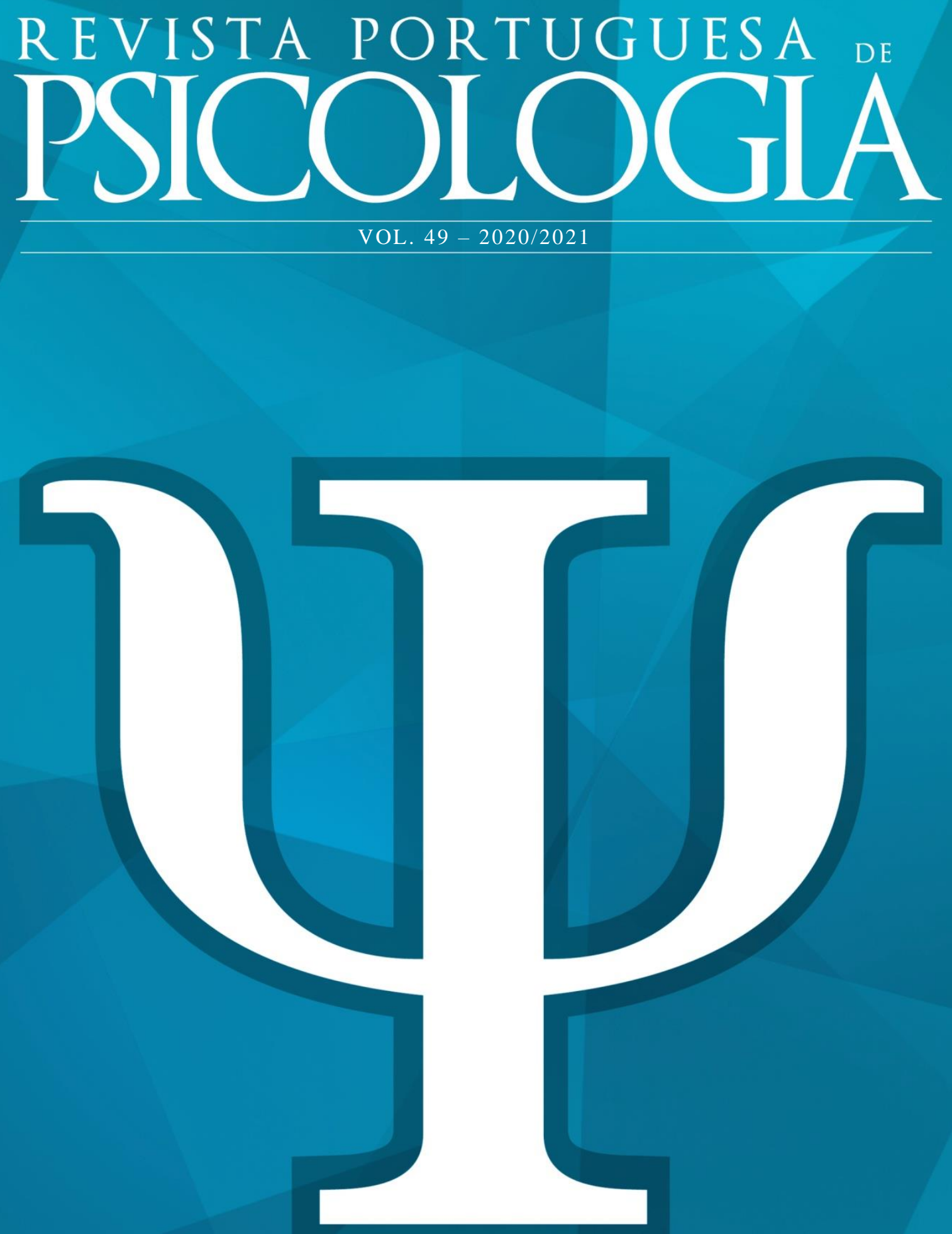


\section{Conselho Editorial / Editorial Board}

Editor:

João Manuel Moreira

Faculdade de Psicologia, Universidade de Lisboa

Conselho Editorial / Editorial Board:

Adelina Lopes da Silva

Faculdade de Psicologia, Universidade de Lisboa

Alexandra Reis

Faculdade de Ciências Humanas e Sociais, Universidade do Algarve

Amâncio da Costa Pinto

Faculdade de Psicologia e CE, Universidade do Porto

Ana Margarida Veiga Simão

Faculdade de Psicologia, Universidade de Lisboa

Bárbara Figueiredo

Escola de Psicologia, Universidade do Minho

Danilo Silva

Faculdade de Psicologia, Universidade de Lisboa

Félix Neto

Faculdade de Psicologia e CE, Universidade do Porto

Helio Carpinteiro

Universidade Complutense de Madrid

Isabel Sá

Faculdade de Psicologia, Universidade de Lisboa

Isabel Soares

Escola de Psicologia, Universidade do Minho

Jean Guichard

Conservatoire National des Artes et Métiers

José Keating

Escola de Psicologia, Universidade do Minho

José Tomás da Silva

Faculdade de Psicologia e CE, Universidade de Coimbra

Leonel Garcia-Marques

Faculdade de Psicologia, Universidade de Lisboa

Leonor Cardoso

Faculdade de Psicologia e CE, Universidade de Coimbra

Luísa Barros

Faculdade de Psicologia, Universidade de Lisboa

Luísa Morgado

Faculdade de Psicologia e CE, Universidade de Coimbra

Manuel Rafael

Faculdade de Psicologia, Universidade de Lisboa

Maria do Céu Taveira

Escola de Psicologia, Universidade do Minho

Maria Eduarda Duarte

Faculdade de Psicologia, Universidade de Lisboa
Maria José Chambel

Faculdade de Psicologia, Universidade de Lisboa

Mário Ferreira

Faculdade de Psicologia, Universidade de Lisboa

Mário Simões

Faculdade de Psicologia e CE, Universidade de Coimbra

Mark Savickas

Northeast Ohio Medical University

Paulo Ventura

Faculdade de Psicologia, Universidade de Lisboa

Pedro B. Albuquerque

Escola de Psicologia, Universidade do Minho

Rita Francisco

Fac. de Ciências Humanas, Univ. Católica Portuguesa

Rocío Fernandez-Ballesteros

Universidad Autónoma de Madrid

Rosário Lima

Faculdade de Psicologia, Universidade de Lisboa

Rui Abrunhosa Gonçalves

Escola de Psicologia, Universidade do Minho

Salomé Vieira Santos

Faculdade de Psicologia, Universidade de Lisboa

São Luís Castro

Faculdade de Psicologia e CE, Universidade do Porto

\section{Assistente Editorial / Editorial Assistant:}

Rita Monteiro / Inês Dias

Editores e Directores Eméritos / Emeriti Editors and Directors:

Henrique Barahona Fernandes

Director, 1967-1991

Agostinho Pereira

Editor, 1967-1969

José Luiz Simões da Fonseca

Editor, 1969-1971

José H. Ferreira-Marques

1992-2001

Danilo Silva

2002-2005

Manuel Rafael

2006-2011

\section{Instruções aos autores:}

https://sites.google.com/site/revistaportuguesadepsicologia /instrucoes-aos-autores

ISSN 2183-3699 (versão electrónica / electronic version) ISSN 0872-0304 (versão impressa / print version) 


\title{
REVISTA PORTUGUESA

\section{Autocriticismo e tentativas de suicídio em indivíduos com experiência de doença mental}

\author{
Ana Rainha \\ Departamento de Psicologia, Escola de Ciências Sociais, Universidade de Évora \\ Rui C. Campos \\ Departamento de Psicologia, Escola de Ciências Sociais, Universidade de Évora
}

\begin{abstract}
RESUMO
$\mathrm{Na}$ presente investigação examinou-se o papel do autocriticismo, enquanto dimensão da personalidade, no risco de suicídio, avaliado através da presença de pelo menos uma tentativa de suicídio. Participaram 92 indivíduos adultos, com idades entre os 20 e os 65 anos $(M$ $=42.73, S D=12.95)$ divididos por três grupos: 22 indivíduos com perturbação psiquiátrica e que tentaram previamente o suicídio, 39 indivíduos com perturbação psiquiátrica sem tentativa prévia de suicídio e 31 indivíduos da comunidade. Os participantes responderam ao Questionário de Experiências Depressivas - versão reduzida - e ao Inventário Breve de Sintomas. Os grupos foram comparados utilizando uma ANOVA e uma ANCOVA, considerando o nível de psicopatologia como covariável. Os resultados indicam que, em ambos os casos, os indivíduos que tentaram previamente o suicídio e os indivíduos com perturbação psiquiátrica sem tentativas prévias de suicídio apresentaram níveis de autocriticismo significativamente mais elevados que os indivíduos da comunidade. Não foram encontradas diferenças significativas entre os dois grupos clínicos.
\end{abstract}

Palavras-chave: Autocriticismo, tentativas de suicídio, risco de suicídio, sintomas psicopatológicos.

\begin{abstract}
This research examined the role of self-criticism as a personality dimension in suicide risk, assessed through the presence of at least one suicide attempt. Ninety-two adults participated, ranging in age from 20 to 65 years $(M=$ 42.73, $S D=12.95$ ), divided into three groups: 22 individuals with a psychiatric disorder and who previously attempted suicide, 39 individuals with a psychiatric disorder without a prior suicide attempt and 31 individuals from the community. Participants answered the Depressive Experiences Questionnaire - Short form - and the Brief Symptom Inventory. The groups were compared using an ANOVA and an ANCOVA, considering psychopathology as a co-variable. Results indicate that individuals who have attempted suicide and individuals with a psychiatric disorder without previous suicide attempts presented significantly higher levels of selfcriticism compared to community individuals. No significant differences were found between the clinical groups.
\end{abstract}

Keywords: Self-criticism, suicide attempts, suicide risk, psychopathological symptoms.

O suicídio é um importante problema de saúde mental ao longo do ciclo vital, quer em populações clínicas quer em populações não clínicas (Campos \& Holden, 2014; Sampaio, 1992, 2002; Saraiva, 1998, 2014), com custos pessoais, sociais e económicos elevados (Chishti et al., 2003; Cordeiro, 2016; Direção Geral de Saúde, 2013; World Health Organization, 2012). De acordo com a World Health Organization (WHO, 2021), mais de 700 mil pessoas morrem por suicídio todos os anos. Em Portugal, segundo dados do Instituto Nacional de Estatística (INE, 2018), em 2016 registou-se um total de 981 mortes por suicídio (9.5 óbitos por 100.000 habitantes). 
Dada a dimensão do fenómeno, compreender as variáveis potencialmente influentes para o risco suicidário constitui um imperativo de saúde pública e uma tarefa de investigação relevante (Campos \& Holden, 2014; Harrison, Geddes, \& Sharpe, 2002; World Health Organization, 2012). Vários modelos teóricos procuraram explorar os fatores de risco para o suicídio e os comportamentos suicidários, entre as quais perspetivas psicológicas e socioculturais. Os fatores de risco costumam diferenciar-se em fatores predisponentes (tipo traço) e fatores desencadeantes ou precipitantes (tipo estado; Hawton \& Van Heeringer, 2009). Entre os fatores psicológicos predisponentes, refira-se a presença de traços de personalidade disfuncionais, que podem interagir com acontecimentos de vida negativos (e.g., Campos, Holden, Caçador, Fragata, \& Baleizão, 2018). Evidência clínica e empírica considerável indica que o autocriticismo e o perfecionismo constituem importantes fatores de vulnerabilidade para o risco de suicídio (Blatt, 2004, 2008; Campos \& Holden, 2014; O’Connor \& Noyce, 2008).

O autocriticismo é uma dimensão de personalidade caracterizada por uma ênfase em questões de autodefinição, controlo, autonomia e, ainda, numa tendência para um escrutínio marcado e contínuo sobre o self, associado a elevados padrões pessoais (Blatt, 2004, 2008; Blatt \& Schichman, 1983). Constitui, paralelamente à dependência/necessidade, uma dimensão proposta no Modelo de Duas Polaridades de Sidney Blatt (2008), que afirma que um desenvolvimento harmonioso da personalidade implica uma progressão sinergética entre dois processos/tarefas desenvolvimentais: o relacionamento e a autodefinição. Na patologia, a ênfase é colocada num dos processos, em detrimento de outro, constituindo-se assim uma vulnerabilidade autocrítica/introjetiva ou dependente/anaclítica, consoante a ênfase é posta na autodefinição ou no relacionamento, respetivamente (Blatt, 2008; Blatt et al., 1982). Com efeito, os indivíduos autocríticos são muito exigentes consigo próprios e podem responder a acontecimentos de vida stressantes com sentimentos de culpa, desespero, vergonha, tendendo a não procurar suporte social como mecanismo para lidar com o sofrimento. Tendem a dirigir a raiva e a hostilidade para os outros de modo a preservar a autoestima (Ashby, Rice, \& Martin, 2006), que é "lábil", dependendo das suas realizações e sucessos pessoais, flutuando entre sentimentos de grandiosidade, superioridade e omnipotência, e sentimentos de falha, fracasso, inferioridade e insucesso (Luyten et al., 2007; Mongrain, 1998).

Diversos estudos empíricos têm demonstrado que existe uma forte relação entre esta dimensão autocrítica da personalidade e a psicopatologia em geral, e a depressão, em particular (Campos, Besser, \& Blatt, 2010; KopalaSibley et al., 2014; Luyten et al., 2007). Os indivíduos autocríticos são particularmente vulneráveis a acontecimentos que ameaçam a sua autoestima e um sentido positivo do self, mas também, a situações de rejeição e abandono (Blatt \& Zuroff, 1992; Campos, 2009, 2012; Luyten \& Blatt, 2011, 2013; Sobrinho \& Campos, 2016). Para além de vulnerabilidade à depressão, o autocriticismo pode constituir uma fonte de vulnerabilidade para uma variedade de outras psicopatologias, como a perturbação da ansiedade social e a perturbação da personalidade borderline (e.g., Kopala-Sibley et al., 2014).

Diferentes investigações têm indicado que o autocriticismo pode ter igualmente um importante papel no risco suicidário (Blatt et al., 1982; Campos, Besser, Abreu, Parreira, \& Blatt, 2014; Campos, Besser, \& Blatt, 2012, 2013; 
Campos \& Holden, 2014, 2015; Campos, Holden, Caçador, Fragata, \& Baleizão, 2018; Fazaa \& Page, 2003, 2009; Klomek et al., 2008; O’Connor \& Noyce, 2008).

Fazaa \& Page (2003), por exemplo, num estudo levado a cabo com uma amostra de estudantes universitários, mostraram que os indivíduos autocríticos que tentaram o suicídio manifestaram uma forte intencionalidade na sua tentativa, obtendo pontuações elevadas numa medida de letalidade. Os indivíduos autocríticos envolveram-se igualmente em gestos suicidas frequentemente em resposta a um stressor intrapsíquico, quer dizer, um acontecimento de vida pessoal que constituiu o "gatilho" para a tentativa de suicídio, devido a uma necessidade urgente de fuga. Também Klomek et al. (2008) analisaram a relação entre suicidalidade e a dimensão de personalidade autocrítica numa amostra de 96 adolescentes. Foram comparados 3 grupos: 32 adolescentes que tentaram o suicídio, 32 com perturbação psiquiátrica e 32 adolescentes sem queixa. Concluíram que os adolescentes que tentaram o suicídio apresentaram níveis significativamente mais elevados de autocriticismo, comparativamente aos dois grupos de controlo. Em Portugal, os resultados de alguns estudos salientaram o papel do autocriticismo para o risco suicidário (e.g., Campos, Besser, Abreu, Parreira, \& Blatt, 2014; Campos, Besser, \& Blatt, 2012; Campos \& Holden, 2015). Contudo, estes estudos foram realizados com amostras não clínicas da comunidade, não tendo estudado especificamente as tentativas de suicídio ou, em alguns casos, não tendo controlado a presença de psicopatologia. Campos, Besser, \& Blatt (2012), por exemplo, examinaram numa amostra de adultos da comunidade a relação entre autocriticismo e suicidalidade, avaliando o distress como variável mediadora. A suicidalidade foi aqui medida enquanto variável latente. Concluíram que o distress mediou a relação entre autocriticismo e suicidalidade. Na sequência destes estudos, seria importante avaliar se o autocriticismo se relaciona com a presença de tentativas de suicídio numa amostra clínica, controlando os níveis de distress dos participantes.

\section{Objetivos do estudo}

Dado que os estudos em Portugal que relacionaram o autocriticismo com o risco suicidário o fizeram com amostras comunitárias, não se focando especificamente nas tentativas de suicídio, o presente estudo teve como objetivo avaliar o papel do autocriticismo no risco de suicídio, avaliado pela presença de pelo menos uma tentativa de suicídio, utilizando uma amostra clínica. Assim, compararam-se indivíduos com perturbação psiquiátrica e que tentaram previamente o suicídio, indivíduos com perturbação psiquiátrica sem tentativa prévia de suicídio e indivíduos da comunidade. Controlou-se também, estatisticamente, o efeito da intensidade da sintomatologia psicopatológica, reduzindo assim a sua influência nos níveis de autocriticismo. Globalmente, os níveis de distress podem influenciar os níveis de autocriticismo (veja-se Campos et al., 2012, 2014). Espera-se que os grupos apresentem diferenças significativas no nível de autocriticismo, com os indivíduos que tentaram o suicídio a apresentarem níveis significativamente mais elevados de autocriticismo comparativamente aos indivíduos com perturbação psiquiátrica sem tentativa de suicídio e aos indivíduos da comunidade. Espera-se que estes efeitos se mantenham, mesmo quando se controla o efeito da psicopatologia. 


\section{MÉTODO}

\section{Participantes e procedimento}

Um total de 92 indivíduos com idades compreendidas entre os 20 e 65 anos, $M=42.73, S D=12.95$, 24 do género masculino e 68 do género feminino, a maioria residentes no distrito de Évora, participaram no estudo. O nível educacional dos participantes variou entre 4 e 17 anos de escolaridade $(M=10.83, S D=3.99)$, com dois participantes a terem menos de 4 anos de escolaridade. Aproximadamente 24\% dos participantes estavam desempregados e $41.3 \%$ não eram casados nem viviam em união de facto. Os 92 participantes foram distribuídos por três grupos: grupo de indivíduos com perturbação psiquiátrica que tentaram previamente o suicídio, grupo de indivíduos com perturbação psiquiátrica sem tentativas prévias de suicídio e grupo de indivíduos da comunidade. As características sociodemográficas e clínicas dos participantes do estudo encontram-se descritas no Quadro 1 (secção Resultados).

O grupo de indivíduos que tentaram previamente o suicídio é composto por pacientes que foram admitidos na consulta externa de psiquiatria no Departamento de Psiquiatria e Saúde Mental do Hospital Espírito Santo de Évora (HESE), alguns deles após a entrada no serviço de urgência devido a tentativa de suicídio. O grupo de controlo de indivíduos com perturbação psiquiátrica sem tentativa prévia de suicídio $(N=39)$ foi igualmente seccionado no Departamento de Psiquiatria do Hospital Espírito Santo de Évora. Nenhum destes participantes tem história de tentativa de suicídio. O grupo de indivíduos adultos da comunidade $(N=31)$ é constituído por pessoas que não apresentavam diagnóstico de perturbação mental e foram recrutados em locais públicos, sendo convidados a participar numa investigação sobre variáveis de personalidade e comportamentos de risco.

Este estudo foi aprovado pela Comissão de Ética da Universidade de Évora, no que respeita à recolha de dados dos indivíduos da comunidade, e pela Comissão de Ética do HESE no que respeita à recolha de dados junto dos pacientes. No caso dos grupos clínicos, todos os protocolos foram recolhidos em sessões individuais por psicólogos estagiários. Os participantes responderam aos questionários, de acordo com um procedimento de autoadministração e, no caso de dois indivíduos com menos de 4 anos de escolaridade, ambos do grupo com tentativa prévia de suicídio, ou em todos os casos que apresentassem dificuldades de leitura e compreensão dos itens, os questionários foram heteroadministrados. Os processos dos pacientes foram consultados para recolha de informação clínica. A recolha de dados no grupo da comunidade foi realizada por assistentes de investigação previamente preparados para a aplicação de questionários. Os protocolos de investigação eram compostos por um conjunto diverso de questionários utilizados noutros estudos. Todos os participantes do estudo assinaram um termo de consentimento informado depois de explicada a natureza da investigação. A participação foi anónima, voluntária e não renumerada. 


\section{Instrumentos de medida}

Ficha de Dados Demográficos e Clínicos: Pedia informações relativamente a variáveis sociodemográficas, como a idade, género, estado civil, nacionalidade, escolaridade, profissão e agregado familiar. Adicionalmente, era pedida informação complementar respeitante a dados clínicos e familiares. No caso dos grupos clínicos, os processos dos pacientes foram consultados para recolha de informação clínica.

Questionário de Experiências Depressivas (QED; Depressive Experience Questionnaire; Blatt et al., 1976, 1979; versão reduzida de Bagby et al., 1994): O QED é um questionário composto por 66 itens, que foi construído para avaliar um conjunto de experiências relacionadas com a depressão, mas que não constituam em si sintomas clínicos de perturbação depressiva (Campos, 2009, 2016). Ao longo do tempo, verificou-se a capacidade do instrumento para avaliar, mais globalmente, as características dependentes e autocríticas da personalidade (Campos, Besser, \& Blatt, 2013). Os indivíduos respondem numa escala de resposta de Likert de sete pontos, variando de 1 - "discordo totalmente" a 7 - “concordo totalmente” (Blatt et al., 1976; Campos, 2016; Campos, Besser, \& Blatt, 2013). Permite obter resultados para três fatores: dependência, autocriticismo e eficácia (Blatt, D’Afflitti, \& Quinlan, 1976, 1979). A consistência interna, a fiabilidade teste-reteste e a validade fatorial do QED têm sido demonstradas em várias investigações e em diferentes culturas (veja-se Campos, 2015, 2017). A versão portuguesa do QED revela propriedades psicométricas adequadas (Campos, 2015, 2017). Os dados relativos à consistência interna e à validade da versão portuguesa do QED foram muito semelhantes aos obtidos com a versão original americana. Devido aos problemas que alguns autores têm salientado na versão original de 66 itens (Desmet et al., 2007), diversas formas reduzidas foram desenvolvidas, nomeadamente a que foi utilizada neste estudo, a forma de 19 itens (Bagby et al., 1994). Acresce que dada a natureza da amostra e o facto de ser necessário aplicar diversos instrumentos de medida, optou-se por uma forma com menos itens, logo com um tempo de resposta bastante mais reduzido. A investigação tem demonstrado a validade desta versão reduzida do QED (Desmet et al., 2007). Na presente investigação, utilizouse apenas o resultado da escala autocriticismo, composta por 10 itens. $\mathrm{O}$ alfa de Cronbach para esta escala foi de .74 .

Inventário Breve de Sintomas Psicopatológicos (BSI; Brief Symptom Inventory; Derogatis, 1993; versão portuguesa de Canavarro, 1999, 2007): O BSI é um instrumento de autorelato de quantificação de sintomas psicopatológicos (Derogatis, 1993). É constituído por 53 itens. Os indivíduos respondem numa escala de resposta tipo Likert de 5 pontos, de 0 - "nunca" a 4 - "muitíssimas vezes", relativa ao grau em que experienciaram cada sintoma na semana anterior. Valores elevados são indicadores de um nível mais elevado de distress. Avalia 9 dimensões sintomáticas e permite a obtenção de três índices globais de distress: Índice Geral de Sintomas (IGS), Índice de Sintomas Positivos (ISP) e Total de Sintomas Positivos (TSP). A fiabilidade e a validade do BSI têm sido apoiadas por múltiplos estudos (Derogatis \& Melisaratos, 1983). Na versão original, os valores alfa de Cronbach variaram entre 71 para a dimensão psicoticismo e .85 para a depressão (Derogatis \& Melisaratos, 1983). A versão portuguesa do inventário (Canavarro, 1999, 2007) apresenta propriedades psicométricas aceitáveis. Os valores alfa de Cronbach assumiram valores aceitáveis de consistência interna, variando entre .70 e .80, à exceção das escalas de ansiedade fóbica e de 
psicoticismo que apresentaram valores ligeiramente inferiores (.62 para ambas as dimensões; Canavarro, 2007). Neste estudo, foi utilizado o Índice Geral de Sintomas (IGS). O valor alpha de Cronbach foi de .98 para a escala total.

\section{Procedimentos de análise de dados}

Num primeiro momento, foi utilizada a análise de variância univariada para avaliar o efeito do tipo de grupo (indivíduos que tentaram previamente o suicídio, indivíduos com perturbação psiquiátrica sem tentativa prévia de suicídio e indivíduos da comunidade) nos níveis de autocriticismo. Realizou-se uma ANOVA a um fator, com Efeitos Fixos (Tipo I) com três níveis do fator que foram fixados a priori. Realizou-se igualmente o teste post-hoc de Bonferroni (Maroco, 2010; Pestana \& Gageiro, 2003). O pressuposto da normalidade das distribuições da variável autocriticismo nos diferentes grupos foi avaliado pelo teste de Kolmogorov-Smirnov (K-S) com correção de Lilliefors e pelo teste de Shapiro-Wilk. Para todos os grupos, a distribuição da variável autocriticismo foi relativamente normal $(p>.05)$. A simetria [Skewness/Std error ${ }_{\text {skewness }}<1.96$ ] e o achatamento mesocúrtico [Kurtosis/Std error kurtosis $_{2}$ 1.96] foram assegurados. O pressuposto da homocedasticidade de variâncias foi validado pelo teste de Levene $(p>.05)$. Analisou-se também a eventual existência de outliers significativos. Verificou-se apenas a existência de um outlier moderado no grupo da comunidade.

Num segundo momento, foi usada a análise de covariância (ANCOVA) para avaliar a relação direta entre o fator e a variável de personalidade, ao controlar o efeito da psicopatologia. A introdução da covariável psicopatologia no modelo permitiu remover, ainda que parcialmente, a sua influência nos níveis de autocriticismo, passando cada um dos grupos a assumir o mesmo nível de psicopatologia global que resultou da sua média $(M=1.34)$. Foi realizada uma ANCOVA para comparar os três grupos relativamente à variável autocriticismo, com a introdução da psicopatologia como variável concomitante, seguida dos testes post-hoc de comparações múltiplas com as médias ajustadas de Bonferroni. O pressuposto da existência de associação linear entre a covariável e a variável dependente foi analisado pela correlação de Bravais-Pearson. A correlação entre a covariável psicopatologia e a variável autocriticismo foi positiva e estatisticamente significativa $(R=.73, p<.001)$, sendo que, aproximadamente, $52.71 \%$ da variância dos níveis de autocriticismo foi explicada pela psicopatologia. As correlações entre a variável autocriticismo e a psicopatologia em cada categoria do fator, ou seja, cada um dos três grupos, revelaram que as correlações foram estatisticamente elevadas, positivas e estatisticamente significativas em cada grupo. O pressuposto da homogeneidade das retas de regressão foi avaliado através da análise gráfica das dispersões para cada grupo com a covariável, calculando-se e desenhando-se a reta de regressão para cada gráfico. Verificou-se o não paralelismo das retas de regressão, sendo que a regressão da variável dependente (autocriticismo) na covariável psicopatologia não foi a mesma para cada grupo. Para testar ainda o feito de interação entre a psicopatologia e o fator, usou-se como medida de associação o Eta. O valor de eta ${ }^{2}$ foi elevado $\left(0.857^{2}=73.44 \%\right)$, demonstrando que, aproximadamente, $73.44 \%$ da variação dos grupos se deveu à presença da covariável psicopatologia. Contudo, a ANCOVA é relativamente robusta a violações neste pressuposto para modelos com um fator fixo (Pestana \& 
Gageiro, 2003), o que permitiu prosseguir com a análise. O pressuposto da homocedasticidade de variâncias no modelo com a covariável foi validado pelo teste de Levene de Igualdade do Erro de Variâncias $(p>.05)$.

\section{RESULTADOS}

\section{Modelo sem a introdução da covariável sintomas psicopatológicos}

Numa primeira análise, analisou-se se as diferenças no nível de autocriticismo dos três grupos (veja-se Quadro 1) foram ou não estatisticamente significativas. A análise de variância univariada (ANOVA) revelou que o fator Grupo teve um efeito estatisticamente significativo e de elevada dimensão, $F(2,89)=22.84, p<.001, \mathrm{n}_{p}^{2}=.34$, com uma potência estatística de 1.00, sobre o autocriticismo.

De acordo com o teste post-hoc de Bonferroni, as diferenças estatisticamente significativas para o fator ocorreram entre o grupo de indivíduos que tentaram o suicídio e o grupo de participantes da comunidade, IC a 95\% [10.48, 24.147], $p$ <.001, e o grupo de indivíduos com perturbação psiquiátrica sem tentativa de suicídio e o grupo de adultos da comunidade, IC a 95\% [7.06, 18.84], $p$ <.001. Não foram encontradas diferenças estatisticamente significativas entre o grupo de indivíduos que tentaram o suicídio e o grupo de indivíduos com perturbação psiquiátrica sem tentativa de suicídio, IC a 95\% [-2.174, 10.888], $p=.32$. A percentagem de variação dos níveis de autocriticismo explicados pelo fator $\left(\mathrm{R}^{2}\right)$ foi de $33.9 \%$.

\section{Modelo com a introdução da covariável sintomas psicopatológicos}

A análise de covariância (ANCOVA) revelou que tanto o efeito do fator, $F=4.54, p=0.013, \eta_{p}^{2}=.10$, com uma potência estatística de .76 , como o efeito da covariável psicopatologia, $F=47.54, p=0.000, \eta_{\mathrm{p}}{ }^{2}=0.36$ ) com uma potência estatística de 1.000, foram estatisticamente significativos. Com a introdução da covariável no modelo, os testes de comparação múltipla de médias revelaram padrões semelhantes de diferenças entre grupos; mostraram que as diferenças estatisticamente significativas para o fator ocorreram entre os indivíduos que tentaram o suicídio e os indivíduos da comunidade, e o grupo de indivíduos com perturbação psiquiátrica e o grupo da comunidade, $p<.05$. Não foram encontradas diferenças estatisticamente significativas entre os indivíduos que tentaram o suicídio e os indivíduos com perturbação psiquiátrica sem tentativa de suicídio, $p>.05$. O modelo com a introdução da covariável psicopatologia reforçou os resultados anteriores e explicou $55.6 \%\left(R^{2}\right.$ ajustado) da variância dos níveis de autocriticismo ${ }^{1}$.

\footnotetext{
${ }^{1}$ De referir que os grupos diferem significativamente no que respeita aos níveis de uma das cinco variáveis sociodemográficas considerada, o nível de escolaridade. Assim sendo, realizou-se igualmente uma ANCOVA, introduzindo esta variável demográfica como covariável. Os resultados são similares, pelo que, por uma questão de simplificação, não são apresentados no texto principal.
} 
Quadro 1

Caraterísticas sociodemográficas e clínicas (Médias e desvios padrão ou percentagens), resultados na variável em estudo autocriticismo e na covariável sintomas psicopatológicos para os três grupos

\begin{tabular}{|c|c|c|c|c|}
\hline & & $\begin{array}{l}\text { Tentativa de } \\
\text { suicídio } \\
(N=22)\end{array}$ & $\begin{array}{c}\text { Perturbação } \\
\text { Psiquiátrica } \\
\quad(N=39)\end{array}$ & $\begin{array}{l}\text { Comunidade } \\
\qquad(N=31)\end{array}$ \\
\hline \multirow[t]{2}{*}{ Idade } & & 44.14 & 44.54 & 39.45 \\
\hline & & $(12.66)$ & $(12.93)$ & $(12.96)$ \\
\hline Género & $\%$ Mulheres & $86.3 \%$ & $69.2 \%$ & $71.0 \%$ \\
\hline \multirow[t]{2}{*}{ Escolaridade em anos } & & 9.82 & 9.85 & 12.77 \\
\hline & & $(5.03)$ & $(3.83)$ & $(2.46)$ \\
\hline Casado ou em união de facto & Sim & $59.0 \%$ & $69.2 \%$ & $45.2 \%$ \\
\hline Desempregado & Sim & $27.2 \%$ & $25.6 \%$ & $19.4 \%$ \\
\hline \multirow{2}{*}{$\begin{array}{l}\text { Tempo médio desde o início } \\
\text { do acompanhamento em anos }\end{array}$} & & 7.33 & 8.05 & \\
\hline & & $(7.61)$ & $(8.22)$ & \\
\hline \multirow[t]{7}{*}{ Diagnóstico } & Perturbação: & & & \\
\hline & Bipolar & 3 & 4 & \\
\hline & Depressiva & 18 & 21 & \\
\hline & Psicótica & 1 & 2 & \\
\hline & Ansiedade & 0 & 8 & \\
\hline & Traumática & 0 & 2 & \\
\hline & Personalidade & 0 & 2 & \\
\hline \multirow[t]{2}{*}{ Autocriticismo } & & 43.82 & 39.46 & 26.51 \\
\hline & & $(9.72)$ & $(10.29)$ & $(9.94)$ \\
\hline \multirow[t]{2}{*}{ Sintomas psicopatológicos } & & 1.90 & 1.53 & 0.71 \\
\hline & & $(0.67)$ & $(0.83)$ & $(0.53)$ \\
\hline
\end{tabular}




\section{DISCUSSÃO}

Este estudo examinou o papel da variável de personalidade autocriticismo no risco de suicídio, avaliado através da presença de pelo menos uma tentativa de suicídio, controlando o efeito da psicopatologia. Em primeiro lugar, verificou-se se os indivíduos que tentaram o suicídio apresentavam diferenças significativas relativamente aos indivíduos dos grupos com perturbação psiquiátrica sem tentativa de suicídio e dos indivíduos da comunidade na variável autocriticismo, avaliada pela forma reduzida do Questionário de Experiências Depressivas, sem controlar o efeito da variável sintomas psicopatológicos. Os resultados não suportaram integralmente a hipótese colocada. Os indivíduos que tentaram o suicídio apresentaram níveis médios de autocriticismo superiores àqueles observados nos indivíduos com perturbação psiquiátrica sem tentativa de prévia de suicídio, mas as diferenças não foram significativas entre os dois grupos. Porém, os indivíduos que tentaram o suicídio e os outros indivíduos com perturbação psiquiátrica apresentaram níveis significativamente mais elevados de autocriticismo do que os indivíduos da comunidade. Estes resultados são diferentes daqueles encontrados por Klomek et al., (2008) com adolescentes, sendo que, nesse estudo, os adolescentes que tentaram o suicídio apresentaram níveis mais elevados de autocriticismo que os adolescentes dos grupos com perturbação psiquiátrica sem tentativa prévia de suicídio e da comunidade.

Examinou-se de seguida se os indivíduos que tentaram suicídio diferiam significativamente dos grupos com perturbação psiquiátrica sem tentativa de suicídio e da comunidade em autocriticismo, controlando o efeito dos sintomas psicopatológicos. Os resultados revelaram uma vez mais a existência de diferenças significativas entre os grupos para os níveis médios de autocriticismo. Porém, os indivíduos que tentaram o suicídio não apresentaram níveis médios de autocriticismo significativamente superiores aos indivíduos com perturbação psiquiátrica sem tentativas prévias de suicídio. Ainda assim, os dois grupos clínicos diferiram do grupo comunitário relativamente aos níveis médios de autocriticismo, quando se controla o nível de sintomas psicopatológicos.

Os resultados obtidos sugerem que o autocriticismo poderá ser mais um fator de vulnerabilidade para a perturbação mental do que especificamente para o risco de suicídio. Os dois grupos clínicos apresentaram níveis de autocriticismo significativamente superiores aos dos indivíduos do grupo comunitário, mesmo quando se controlou o nível global de sintomas psicopatológicos. Estes resultados estão de acordo com investigações prévias que apontam para que o autocriticismo possa constituir um importante fator de vulnerabilidade para perturbação psicopatológica (e.g., Bers, Besser, Harpaz-Rotem, \& Blatt, 2013; Luyten et al., 2007). Indivíduos com elevado autocriticismo são particularmente vulneráveis, muito provavelmente devido à sua elevada exigência pessoal e ao facto de serem muitas vezes atormentados pela dúvida e introspeção dolorosa e recorrerem pouco aos outros perante situações de stress e exigência (Blatt, 1995, 2008; O`Connor \& Noyce, 2008).

Alternativamente, a ausência de diferenças significativas entre os dois grupos clínicos poderá ser explicada pela ausência de diferenças significativas nos níveis globais de sintomas psicopatológicos entre esses dois grupos. Seria expectável que os indivíduos que tentaram o suicídio (e, portanto, em maior risco de suicídio) apresentassem níveis significativamente mais elevados de psicopatologia quando comparados com os indivíduos com perturbação 
psiquiátrica sem tentativa de suicídio. Note-se também que uma elevada percentagem de participantes no grupo sem tentativa de suicídio apresenta um diagnóstico de depressão (54\%). Este facto pode ajudar a explicar os elevados níveis de autocriticismo verificados neste grupo e a ausência de diferenças significativas para o grupo com tentativa de suicídio.

Note-se que, recorrendo a um estudo anterior (Klomek et al., 2008) e considerando o tamanho do efeito aí obtido para o teste da diferença entre os grupos com e sem tentativa em autocriticismo ( $d$ de Cohen $=1.31$ ), calculou-se a potência de teste para a comparação dos mesmos grupos no nosso estudo com um t-test, tendo em conta a real dimensão dos grupos que participaram, recorrendo ao software G*Power (Faul, Erdfelder, Lang, \& Buchner, 2007). A potência estimada foi de 0.99 . Pode concluir-se que o nível de potência providenciado pela amostra recolhida seria suficiente caso o tamanho do efeito no presente estudo fosse semelhante ao do estudo citado, pelo que a ausência de uma diferença significativa no presente estudo se ficou a dever ao facto de o efeito ser efetivamente menor.

Refira-se ainda que, neste estudo, se utilizou uma versão reduzida do Questionário de Experiências Depressivas (adaptada de Bagby et al., 1994) e não a versão original de 66 itens, sendo que é, tanto quanto é do nosso conhecimento, o primeiro estudo em Portugal que utilizou a versão reduzida. Apesar de alguns estudos apontarem para dificuldades várias da forma original, outros estudos sugerem que poderá ser claramente superior às formas reduzidas na avaliação dos constructos da teoria de Blatt (Zuroff, Mongrain, \& Santor, 2004).

\section{Limitações, conclusões e implicações clínicas}

Este estudo apresenta algumas limitações. Primeiro, recorreu-se a um método de amostragem não probabilístico, sendo que a amostra resultante é pequena e não representativa da população em estudo e os grupos são de diferentes dimensões (design não balanceado). Segundo, utilizou-se uma versão reduzida do Questionário de Experiências Depressivas (QED). Apesar da sua utilidade em amostras com níveis baixos de energia anímica (Desmet et al., 2007), a versão integral do QED com os 66 itens poderá ser superior (Zuroff et al., 2004). Terceiro, foram usadas somente medidas de autorrelato para a recolha de dados. Quarto, nesta investigação foi usado um design transversal que impede o estabelecimento de relações causais entre as variáveis. Quinto, não foram avaliadas outras variáveis potencialmente relevantes e associadas ao risco de suicídio, particularmente acontecimentos de vida negativos, depressão e desesperança. Estudos futuros deveriam replicar os resultados explorando estas limitações.

Apesar das limitações, os resultados obtidos permitem considerar a dimensão autocrítica da personalidade, ainda assim, como um importante fator de vulnerabilidade associado à perturbação mental. Os resultados sugerem que níveis elevados de autocriticismo permitem discriminar amostras clínicas de amostras não clínicas, mesmo quando se controlam os níveis globais de sintomas psicopatológicos. A avaliação clínica do autocriticismo pode ser importante na compreensão da psicopatologia e para implementar planos de intervenção terapêutica mais adequados (Harold et al., 2016). Fica por esclarecer um eventual papel específico do autocriticismo no risco suicidário. Esta influência pode ser apenas indireta, através do seu contributo para a perturbação mental. Na verdade, na prática 
clínica, uma avaliação detalhada dos fatores de risco suicidário contribui para a definição de programas de prevenção primária e secundária do suicídio e comportamentos suicidários, auxiliando no processo de tomada de decisão clínica dos profissionais de saúde ao nível das intervenções a implementar (Direção Geral de Saúde, 2013; Santos et al., 2012; World Health Organization, 2012).

\section{REFERÊNCIAS BIBLIOGRÁFICAS}

Ashby, J. S., Rice, K. G., \& Martin, J. L. (2006). Perfectionism, shame, and depressive symptoms. Journal of Counseling \& Development, 84, 148-156.

Bagby, R. M., Parker, J. D. A., Joffe, R. T., \& Buis, T. (1994). Reconstruction and validation of the Depressive Experiences Questionnaire. Assessment, 1, 59-68.

Bers, S. A., Besser, A., Harpaz-Rotem, I., \& Blatt, S. J. (2013). An empirical exploration of the dynamics of anorexia nervosa: Representations of Self, mother, and father. Psychoanalytic Psychology, 30, 188-209.

Blatt, S. J. (1995). The destructiveness of perfectionism: Implications for the treatment of depression. American Psychologist, $12,1003-1020$.

Blatt, S. J. (2004). Experiences of depression: Theoretical, research and clinical perspectives. Washington, DC: American Psychological Association.

Blatt, S. J. (2008). Polarities of experience: Relatedness and self-definition in personality development, psychopathology and the therapeutic process. Washington, DC: American Psychological Association.

Blatt, S. J., D’Afflitti, J., \& Quinlan, D. (1976). Experiences of depression in normal young adults. Journal of Abnormal Psychology, 85, 383-389.

Blatt, S. J., D`Afflitti, J., \& Quinlan, D. (1979). Depressive Experiences Questionnaire (Unpublished manual). New Haven, CT: Yale University.

Blatt, S. J., Quinlan, D. M., Chevron, E. S., McDonald, C., \& Zuroff, D. (1982). Dependency and self-criticism: Psychological dimensions of depression. Journal of Consulting and Clinical Psychology, 1, 113-124.

Blatt, S. J., \& Shichman, S. (1983). Two primary configurations of psychopathology. Psychoanalysis and Contemporary Thought, 6, 187-254.

Blatt, S. J., \& Zuroff, D. C. (1992). Interpersonal relatedness and self-definition: Two prototypes for depressive clinical. Clinical Psychological Review, 12, 527-562.

Campos, R. C. (2009). Depressivos somos nós: Considerações sobre a depressão, a personalidade e a dimensão depressiva da personalidade. Coimbra: Edições Almedina.

Campos, R. C. (2012). Textos sobre psicopatologia e diagnóstico psicodinâmico. Lisboa: Climepsi.

Campos, R. C. (2016). Questionário de Experiências Depressivas: Manual Técnico - Edição Actualizada e Revista. Évora, Portugal: Centro de Investigação em Educação e Psicologia - Universidade de Évora (CIEP-EU).

Campos, R. C. (2017). Dados psicométricos adicionais da versão portuguesa do Questionário de Experiências Depressivas. Revista Iberoamericana de Diagnóstico y Evaluación e Avaliação Psicológica, 46, 121-131.

Campos, R. C., Besser, A., Abreu, H., Parreira., \& Blatt, S. J. (2014). Personality vulnerabilities in adolescent suicidality: The mediating role of psychological distress. Bulletin of Menninger Clinic, 78, 115-139.

Campos, R. C., Besser, A., \& Blatt, S. J. (2010). The mediating role of self-criticism and dependency in the association between perceptions of maternal caring and depressive symptoms. Depression And Anxiety, 27, 1149-1157. 
Campos, R. C., Besser, A., \& Blatt, S. J. (2012). Distress mediates the association between personality predispositions and suicidality: A preliminary study in a Portuguese community sample. Archives of Suicide Research, 16, 44-58.

Campos, R. C., Besser, A., \& Blatt, S. J. (2013). The Portuguese version of the Depressive Experiences Questionnaire (DEQ): Results from a validation program in clinical and non-clinical samples. Spanish Journal of Psychology, 16, 1-13.

Campos, R. C., \& Holden, R. R. (2014). Suicide risk in a Portuguese non-clinical sample of adults. European Journal Psychiatry, 28, 230-241.

Campos, R. C., \& Holden, R. R. (2015). Testing models relating rejection, depression, interpersonal needs, and psychache to suicide risk in nonclinical individuals. Journal of Clinical Psychology, 1-10.

Campos, R. C., Holden, R. R., Caçador, B., Fragata, A. S., \& Baleizão, C. (2018). Self-criticism, intensity of perceived negative life events, and distress: Results from a two-wave study. Personality and Individual Diferences, 124, 145-149.

Canavarro, M. C. (1999). Inventário de Sintomas Psicopatológicos: BSI. In M. R. Simões M. Gonçalves, \& L. S. Almeida (Vol. Eds), Testes e provas psicológicas em Portugal: II. Testes e provas psicológicas em Portugal (pp.87-109). Braga: SHO/APPORT.

Canavarro, M. C. (2007). Inventário de Sintomas Psicopatológicos (BSI): Uma revisão crítica dos estudos realizados em Portugal. In M. R. Simões, C. Machado, M. M. Gonçalves, \& L. S. Almeida (Eds.), Avaliação Psicológica: Instrumentos Validados para a População Portuguesa (Vol. III, pp. 305-330). Coimbra: Quarteto Editora.

Chishti, P., Stone, D. H., Corcoran, P., Williamson, E., \& Petridou, E. (2003). Suicide mortality in the European Union. European Journal of Public Health, 13, 108-114.

Cordeiro, J. D. (2016). Manual de Psiquiatria Clínica (5ª edição revista e atualizada). Lisboa: Fundação Calouste Gulbenkian.

Desmet, M., Vanheule, S., Groenvynck, H., Verhaeghe, P., Vogel, J., \& Bogaerts, S. (2007). The Depressive Experiences Questionnaire: An inquiry into the different scoring procedures. European Journal of Psychological Assessment, 23, $89-98$.

Derogatis, L. R. (1993). BSI: Administration, scoring, and procedures for the Brief Symptom Inventory ( ${ }^{\text {rd }}$ ed.). Minneapolis, MN: National Computer Systems.

Derogatis, L. R., \& Melisaratos, N. (1983). The Brief Symptom Inventory: An introductory report. Psychological Medicine, 13, 595-605

Direção-Geral de Saúde (2013). Programa nacional de saúde mental: Programa nacional de prevenção do suicídio $2013 / 2017$. Lisboa: Autor.

Faul, F., Erdfelder, E., Lang, A., \& Buchner, A. (2007). G*Power 3: A flexible statistical power analysis program for the social, behavioral, and biomedical sciences. Behavior Research Methods, 39, 175-191

Fazaa, N., \& Page, S. (2003). Dependency and self-criticism as predictors of suicidal behavior. Journal of Suicide \& LiveThreatening Behaviour, 33, 172-185.

Fazaa, N., \& Page, S. (2009). Personality style and impulsivity as determinants of suicidal subgroups. Archives of Suicide Research, 13, 31-45.

Harold, C., Marno, S., Dinger, U., Barnett, M. S., \& Barber, J. (2016). Dependency and self-criticism in treatment for depression. Journal Counseling Psychological, 63, 452-459.

Harrison, P., Geddes, J., \& Sharp. M. (2002). Introdução à psiquiatria. Lisboa: Climepsi Editores.

Hawton, K., \& Van Heeringen, K. (2009). Suicide. The Lancet, 373, 1372-1381.

Instituto Nacional de Estatística (INE). (2018). Causas de morte 2016. Estatísticas oficiais.

Luyten, P., \& Blatt, S. J. (2011). Integrating theory-driven and empirically-derived models of personality development and psychopathology: A proposal for DSMV. Clinical Psychology Review, 31, 52-68. 
Luyten, P., \& Blatt, S. J. (2013). Interpersonal relatedness and self-definition in normal and disrupted personality development: Retrospect and prospect. American Psychologist, 68, 172-183.

Luyten, P., Sabbe, B., Blatt, S. J., Meganck, S., Jansen, B., Grave, C., Maes, F., \& Corveleyn, J. (2007). Dependency and selfcriticism: relationship with major depressive disorder, severity of depression, and clinical presentation. Depression and Anxiety, 24, 586-596.

Maroco, J. (2010). Análise estatística com utilização do SPSS (3 ${ }^{\mathrm{a}}$ Ed.). Lisboa: Edições Sílabo.

Klomek, A. B., Orbach, I., Sher, L., Sommerfeld, E., Diller, R., Apter, A., Shahar, G., \& Zalsman, G. (2008). Quality of depression among suicidal inpatient youth. Archives of Suicidal Research, 12, 133-140.

Kopala-Sibley, D. C., Zuroff, D. C., Russell, J.J., \& Moskowitz, D. S. (2014). Understanding heterogeneity in social anxiety disorder: Dependency and self-criticism moderate fear responses to interpersonal cues. British Journal of Clinical Psychology, 53, 141-156.

Mongrain, M. (1998). Parental representations and support-seeking behaviors related to dependency and self-criticism. Journal of Personality, 2, 151-173.

O'Connor, R. C., \& Noyce, R. (2008). Personality and cognitive processes: Self-criticism and different types of rumination as predictors of suicidal ideation. Behaviour Research and Therapy, 46, 392-401.

Pestana, M. H., \& Gageiro, J. N. (2003). Análise de dados para ciências sociais: A complementaridade do SPSS (3ª Ed). Lisboa: Edições Sílabo.

Sampaio, D. (1992). Ninguém Morre Sozinho - O adolescente e o suicídio. Lisboa: Editorial Caminho.

Sampaio, D. (2002). Lições do Abismo. Lisboa: Editorial Caminho.

Santos, J. C., Façanha, J. D., Gonçalves, M. A., Erse, M. P., Cordeiro, R. A., \& Façanha, R. M. (2012). Guia orientador de boas práticas para a prevenção de sintomatologia depressiva e comportamentos da esfera suicidária. Caderno da Ordem dos Enfermeiros, 4, 1-106.

Saraiva, C. B. (1998). Para-suicídio: Contributo para uma compreensão clínica dos comportamentos suicidários recorrentes. Coimbra: Quarteto Editora.

Saraiva, C. B. (2014). Depressão e suicídio: Um guia clínico nos cuidados de saúde primários. Lisboa: LIDEL

Sobrinho, A. T., \& Campos. (2016). Perceção de acontecimentos de vida negativos, depressão e risco de suicídio em jovens adultos. Análise Psicológica, 34, 47-59.

World Health Organization (2021). News, June,17, 2001. https://www.who.int/news-room/fact-sheets/detail/suicide

Zuroff, D. C., Mongrain, M., \& Santor, D. A. (2004). Conceptualizing and measuring personality vulnerability to depression: Comment on Coyne and Whiffen (1995). Psychological Bulletin, 130, 489-511. 